\title{
Estimation of lipid and water concentrations in scattering media with diffuse optical spectroscopy from 900 to $1600 \mathrm{~nm}$
}

\author{
Rami Nachabé \\ Benno H. W. Hendriks \\ Adrien E. Desjardins \\ Marjolein van der Voort \\ Martin B. van der Mark \\ Philips Research \\ Minimally Invasive Healthcare Department \\ 5656 AE Eindhoven \\ The Netherlands
}

\author{
Henricus J. C. M. Sterenborg \\ Erasmus Medical Center \\ Center of Optical Diagnostics and Therapy \\ Department of Radiation Oncology \\ P.O. Box 5201 \\ 3008 AE Rotterdam \\ The Netherlands
}

\begin{abstract}
We demonstrate a method to estimate the concentrations of water and lipid in scattering media such as biological tissues with diffuse optical spectra acquired over the range of 900 to $1600 \mathrm{~nm}$. Estimations were performed by fitting the spectra to a model of light propagation in scattering media derived from diffusion theory. To validate the method, spectra were acquired from tissue phantoms consisting of lipid and water emulsions and swine tissues ex vivo with a two-fiber probe. $\odot 2010$ Society of Photo-Optical Instrumentation Engineers. [DOI: 10.1117/1.3454392]
\end{abstract}

Keywords: near-infrared spectroscopy; lipid; water; tissue composition.

Paper 09530RR received Nov. 30, 2009; revised manuscript received Apr. 29, 2010; accepted for publication May 3, 2010; published online Jun. 21, 2010.

\section{Introduction}

Diffuse optical spectroscopy (DOS) is a widely used technique that can provide a wealth of clinically relevant information about the physiological composition of tissues. ${ }^{1-4}$ DOS measurements can be obtained noninvasively with an optical fiber probe, making them well-suited for guiding tissue resections during open surgeries. In the field of biomedical spectroscopy, many studies have focused on the estimation of the concentrations of deoxyhemoglobin $(\mathrm{Hb})$ and oxyhemoglobin $\left(\mathrm{HbO}_{2}\right)$. In the visible and near-infrared (NIR) wavelength ranges, these two chromophores are often the dominant absorbers in biological tissue. ${ }^{1-6}$ However, lipid and water concentrations can also be of interest to discriminate between malignant and normal tissues in the breast ${ }^{7}$ and to distinguish benign lesions from cysts in breast ${ }^{8}$ - in particular, when employed in fiber-tissue probes.

The estimation of lipid and water concentrations with DOS in the 900-1000-nm wavelength range was addressed by several studies. ${ }^{9,10}$ These studies focused on measurements on phantoms made of intralipid or emulsions made with different kinds of oils. However, very few studies have focused on the estimation of lipid and water concentrations in biological tissues with DOS with wavelengths above $1000 \mathrm{~nm} .{ }^{11-13}$ This wavelength region is potentially attractive from the standpoint of measuring concentrations of water and lipid because absorption features of these chromophores are more prominent than those in the 900-1000-nm range.

Several methods have been demonstrated for estimating optical properties and chromophore concentrations from DOS measurements. Several models have been widely used over

Address all correspondence to Rami Nachabé, Philips, HighTechCampus 34 Eindhoven, Noord-Brabant 5656AE Netherlands; Tel: 31616911207; E-mail: rami.nachabe@philips.com the last decade, such as analytical solutions derived from diffusion theory, ${ }^{1,14}$ differential path length spectroscopy, ${ }^{6,15}$ combined frequency-domain and continuous wave broadband diffuse optical spectroscopy ${ }^{7,10}$ and empirical models based on Monte Carlo simulations and experimental phantoms with known optical properties. ${ }^{14,16-19}$ However, these studies were applied only to wavelengths ranges below $1000 \mathrm{~nm}$.

The model proposed in this study utilizes an analytical solution of a diffusion theory approximation to light propagation in scattering media. First described by Farrell et al., ${ }^{14}$ it expresses the intensity of received light as a function of the optical properties of the medium-i.e., the absorption and the scattering - and the distance between the emitting source and location at the surface of the medium where it is received. To the authors' knowledge, this study represents the first application of the Farrell model to DOS measurements in the wavelength range of 900 to $1600 \mathrm{~nm}$.

To validate the estimation method, DOS measurements were performed on tissue phantoms consisting of custom emulsions for which the lipid and water content were accurately known. Commercial food products, such as butters and margarine, were also utilized as phantoms. To investigate the accuracy of the algorithm with different scattering properties, the particle size distribution (PSD) was varied. To provide an indication of the relevance of this method for deriving concentrations from biological tissue, spectra were acquired from swine tissue ex vivo.

\section{Materials and Methods}

\subsection{Experimental Setup}

A 1.3-mm-diameter optical probe was used for which the distal end was angled $70 \mathrm{deg}$. The probe contains two optical

1083-3668/2010/15(3)/037015/10/\$25.00 @ 2010 SPIE 


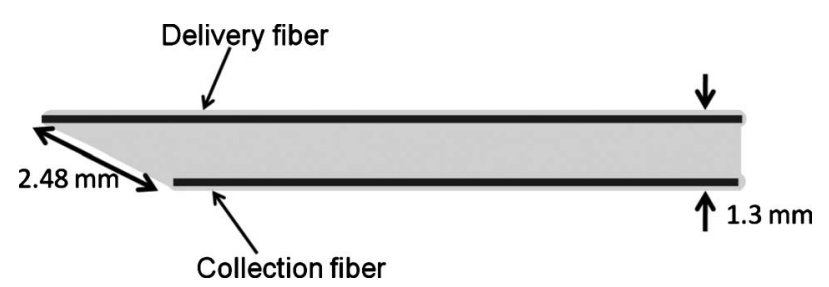

Fig. 1 Schematic sketch of the sagittal cross section of the probe. The distal end is on the left side, and the two lines inside the probe correspond to the axis of symmetry of each of the two optical fibers.

fibers with axis of symmetry parallel to the axis of symmetry of the probe; therefore, the fibers were separated at the distal end by a distance of $2.48 \mathrm{~mm}$ (center to center). Figure 1 shows a sagittal cross section of the probe where the two lines inside the probe correspond to the axis of symmetry of the two optical fibers. The optical fibers are low-OH fibers from Ocean Optics of 220 microns diameter with a core of 200 microns and an NA of 0.22 . One fiber is connected to a tungsten halogen broadband light source with an integrated shutter (Ocean Optics, HL-2000-HP), and the second fiber is connected to a spectrometer with a spectral response from 800 to $1700 \mathrm{~nm}$. The spectrometer has a holographic grating (150 grooves/mm and $1250-\mathrm{nm}$ blaze), an InGaAs sensor array of $512 \times 1$ pixels, and a pixel size of 500 $\times 50$ microns (Andor Technology, DU492A -1.7).

\subsection{Data Preprocessing}

The data was acquired via an interface where the integration time can be set between $1 \mu$ s and several minutes. To suppress the dark current, the detector is thermoelectrically cooled to a temperature of $-50{ }^{\circ} \mathrm{C}$. A wavelength calibration was performed to assign a wavelength value to each pixel of the detector. This was done by fitting a second-order polynomial to a set of atomic lines from an argon light source with peaks at known wavelengths.

The spectra $S\left(\lambda_{i}\right)$ that were processed to estimate the amount of lipid and water were calibrated by first acquiring an intensity calibration spectrum $C\left(\lambda_{i}\right)$. To this aim, the reflectance of a white reflectance standard (LabSphere, WS-1-SL) was measured. A custom-made probe holder was made to hold the probe tightly and keep the surface of the optical fibers at the distal end parallel to the reflectance standard at a fixed distance of $2 \mathrm{~mm}$. The white reflectance standard reflects the light uniformly over the probe surface, and this spectrum was used as the system response to compensate for the spectral shape of the light emitted by the lamp and the wavelengthdependent sensitivity of the detector as well as any wavelength-dependent sensitivity in the optics and gratings of the system. This calibration step was followed by a background measurement in order to minimize the impact of the ambient light, dark current, and electric offsets of the detector. As soon as the detector temperature was stable and the light output was stable (roughly $5 \mathrm{~min}$ after starting our setup), the calibration was performed before each set of measurements.

The spectrum of the tissue can be described as a function of wavelength with the following equation:

$$
S\left(\lambda_{i}\right)=\frac{T\left(\lambda_{i}\right)-T_{b g}\left(\lambda_{i}\right)}{C\left(\lambda_{i}\right)-C_{b g}\left(\lambda_{i}\right)},
$$

where $S\left(\lambda_{i}\right), T\left(\lambda_{i}\right)$, and $C\left(\lambda_{i}\right)$ are the calibrated tissue spectrum, the effective measured tissue spectrum, and the white reflectance standard measured spectrum, respectively. After each tissue measurement, a background measurement $T_{b g}\left(\lambda_{i}\right)$ is acquired by shuttering the light input and subtracted from $T\left(\lambda_{i}\right)$. A background measurement $C_{b g}\left(\lambda_{i}\right)$ is also acquired after the measurement of the white reflectance standard $C\left(\lambda_{i}\right)$.

All the measurements discussed throughout this paper were taken by placing the surface of the optical fibers at the distal end parallel and in contact with the phantoms and the tissues. Having polished fibers at an angle and the size of the optical fiber surface did not hamper our parameter estimation since the distance between the emitting and collecting fibers was sufficiently large (i.e., $2.48 \mathrm{~mm}$ ) that it did not influence our spectral fitting. For much shorter fiber distance separation, the effective radius of the optical fibers and the probe of the geometry influence the reflectance measurements and thus should be taken into account. ${ }^{17,19}$

\subsection{DOS Model and Data Analysis}

Several models ${ }^{14,16-20}$ have been described in the literature to express the intensity of light collected by a fiber after several scattering and absorption events in a diffuse medium. The model that is used in this paper is expressed in Eq. (2) and corresponds to the solution of the diffusion equation for a semi-infinite medium and is a widely accepted model in the field of biomedical photonics. ${ }^{14}$ This analytical expression for the diffuse reflectance $R\left[\mu_{a}(\lambda), \mu_{s}^{\prime}(\lambda), \rho\right]$ is a function of the distance $\rho$ between the emitting and collecting fiber, the absorption coefficient $\mu_{a}(\lambda)$, and the reduced scattering coefficient $\mu_{s}^{\prime}(\lambda)$ and is given by $R\left[\mu_{a}(\lambda), \mu_{s}^{\prime}(\lambda), \rho\right]$ :

$$
\begin{aligned}
& R\left[\mu_{a}(\lambda), \mu_{s}^{\prime}(\lambda), \rho\right] \\
& \quad=\frac{\mu_{s}^{\prime}}{4 \pi\left(\mu_{s}^{\prime}+\mu_{a}\right)}\left[z_{0}\left(\mu_{e f f}+\frac{1}{\widetilde{r}_{1}}\right) \frac{\exp \left(-\mu_{e f f} \widetilde{r}_{1}\right)}{\widetilde{r}_{1}^{2}}\right. \\
& \left.\quad+\left(z_{0}+2 z_{b}\right)\left(\mu_{e f f}+\frac{1}{\widetilde{r}_{2}}\right) \frac{\exp \left(-\mu_{e f f} \widetilde{r}_{2}\right)}{\widetilde{r}_{2}^{2}}\right],
\end{aligned}
$$

where $\mu_{e f f}=\left[3 \mu_{a}\left(\mu_{a}+\mu_{s}^{\prime}\right)\right]^{1 / 2}$ is the effective attenuation coefficient and $z_{0}=\left(\mu_{a}+\mu_{s}^{\prime}\right)^{-1}$ is the location of the virtual scattering source. The extrapolated boundary condition is expressed as $z_{b}=2 A D$, where $A$ is a parameter that depends on the refractive index of the tissue and the surrounding medium. An analytical model for $A$ was described in Ref. 14 that expresses $A$ as a function of the relative refractive index and the critical angle. We made the assumption that the difference in refractive index of tissue and the optical fibers is small and therefore considered that there was no refractive index mismatch, which leads to setting $A=1$. Furthermore, $D=\left[3\left(\mu_{a}\right.\right.$ $\left.\left.+\mu_{s}^{\prime}\right)\right]^{-1}$ is the diffusion constant, $\widetilde{r}_{1}=\left(z_{0}^{2}+\rho^{2}\right)^{1 / 2}$ is the distance between the single scattering source and the collecting fiber, and $\widetilde{r}_{2}=\left[\left(z_{0}+2 z_{b}\right)^{2}+\rho^{2}\right]^{1 / 2}$ is the distance between the image source and the collecting fiber. In this model, the reduced scattering coefficient $\mu_{s}^{\prime}=\mu_{s}(1-g)$ is a function of the scattering coefficient $\mu_{s}$ and the anisotropy parameter $g$. 
However, the reduced scattering parameter can be approximated by a power law function as $\mu_{s}^{\prime}=a\left(\lambda / \lambda_{0}\right)^{-b}$ with amplitude $a$ and where $b$ is a parameter related to the particle size. ${ }^{4}$ Here, $\lambda_{0}$ is a normalization wavelength that is set to $1200 \mathrm{~nm}$, and thus $a$ corresponds to the reduced scattering at this specific wavelength $\left[a=\mu_{s}^{\prime}\left(\lambda_{0}=1200 \mathrm{~nm}\right)\right]$. The absorption coefficient is expressed as

$$
\mu_{a}(\lambda)=f_{\text {Water }} \cdot \mu_{a}^{\text {Water }}(\lambda)+f_{\text {Lipid }} \cdot \mu_{a}^{\text {lipid }}(\lambda),
$$

where $f_{\text {Water }}$ and $f_{\text {Lipid }}$ are the water and lipid volume fractions, respectively, and $\mu_{a}^{\text {Water }}(\lambda)$ and $\mu_{a}^{\text {Lipid }}(\lambda)$ are the absorption coefficients of water and lipid, respectively.

Data were analyzed by fitting Eq. (2) to the processed measurement data obtained by Eq. (1) in order to estimate the optical properties of the probed samples. The parameter $\rho$ that corresponds to the distance between the emitting and collecting fiber is a fixed input argument set at $2.48 \mathrm{~mm}$. Equations (1) and (2) are related by a wavelength-independent constant. This constant corresponds to the ratio between the fraction of light from the calibration standard collected by the fiber and the light from the measured phantoms. The fitting locks onto the spectral shape of the measurements, while the constant, which is also derived from the fitting, accounts for the absolute value of the fitting. The fitting procedure of the spectrum is a Levenberg-Marquardt minimization method available in MATLAB, which produced an estimation of the parameters $f_{\text {Water }}, f_{\text {Fat }}, a$, and $b$. The advantage of this way of fitting is that the absorption coefficients of pure water and pure lipid are input to the model that constrains the spectral shape. The following section describes in detail how we obtained, from measured spectra, these two wavelength-dependent absorption coefficients that are essential for the fitting procedure. Moreover, the reduced scattering coefficient is defined by the amplitude parameter $a$ and slope parameter $b$ only. In contrast, Refs. 7 and 10 fit Eq. (3) to prefitted absorption and reduced scattering or measured optical properties for a set of wavelengths.

In order to assess the reliability of the estimated parameters, the goodness of fit was assessed with the covariance matrix. $^{21}$

The model holds when the reduced scattering is greater than the absorption and the distance between the optical fibers is greater than the scattering length. Moreover, the medium should be homogenous. In this paper, our phantoms and tissue measurements fulfill these assumptions to some extent.

\subsection{Absorption Measurement of Lipid and Water}

It should be emphasized that accurate tables of extinction coefficients as a function of wavelength are required to estimate the exact amount of chromophores. ${ }^{15}$ In order to ensure the optimization of the fits to the model, we decided to measure our own absorption spectra of water and lipid in the wavelength range of 900 to $1600 \mathrm{~nm}$.

The absorption properties of lipid and water were computed based on transmission measurements at constant temperature of $30{ }^{\circ} \mathrm{C}$ through cuvettes of $0.1,0.2,0.5$, and $1 \mathrm{~cm}$ width with a 1-nm resolution spectrophotometer (Lambda 900 Spectrometer, Perkin Elmer). These measurements were made at different temperatures ranging from $30{ }^{\circ} \mathrm{C}$ up to $45^{\circ} \mathrm{C}$ to assess the dependence of the absorption values to temperature. It appeared that there were no changes in the absorption of lipid. In the case of water, the higher the temperature, the more the 972-nm absorption peak of the water shifts to lower wavelengths (roughly $5 \mathrm{~nm}$ for an increase of temperature from 30 to $40{ }^{\circ} \mathrm{C}$ ), resulting in a higher absorption value (5\% relative increase of the absolute value). Additionally, it appeared that the water absorption at $1192 \mathrm{~nm}$ decreases with negligible shift of the peak. Figures 2(a) and 2(b) show the absorption coefficient of water at 30,34, 37, and $40{ }^{\circ} \mathrm{C}$ for different wavelengths ranges. (A linear scale was applied to the figure to highlight the differences.)

Figure 3 depicts the absorption coefficients of water $\mu_{a}^{\text {Water }}(\lambda)$ and lipid $\mu_{a}^{\text {Fat }}(\lambda)$ between 900 and $1600 \mathrm{~nm}$ at $30{ }^{\circ} \mathrm{C}$. The type of lipid that was used was $100 \%$ beef fat. The absorption peaks at 930, 1040, 1211, 1392, and $1413 \mathrm{~nm}$ are in good agreement with measurements performed by Van Veen et al. ${ }^{22}$ in the visible-NIR range and Anderson et al. in the NIR-mid-IR range. ${ }^{11}$ The water absorption peaks at 972 , 1192 , and $1453 \mathrm{~nm}$ are also in good agreement with the measurements reported by Hale and Querry. ${ }^{23}$ In addition, the absolute value of the measured absorption of the lipid and water is in good agreement with that published by Anderson et al. ${ }^{11}$

\subsection{Tissue Phantom Preparation}

Phantoms were made by mixing three ingredients: $100 \%$ beef fat (commercial frying fat), water, and an emulsifier (Triton X-100 from Roche). According to the technical data sheet of the commercial frying fat, the solid fraction compositions (SFC) were respectively $37 \%$ at $20{ }^{\circ} \mathrm{C}, 20 \%$ at $30^{\circ} \mathrm{C}$, and $4 \%$ at $40{ }^{\circ} \mathrm{C}$. The beef fat was melted by heating it to a temperature such that the SFC is less than $1 \%$ to make it completely liquid and thus easy to mix with water. Taking this into consideration, the water, the lipid, and the emulsifier were heated to $50{ }^{\circ} \mathrm{C}$, below the cloud temperature of the Triton $\mathrm{X}-100$, which is $65^{\circ} \mathrm{C}$ and high enough to get a liquid lipid. The amount of emulsifier comprised $4 \%$ of the total amount of lipid that was used to prepare the phantoms as suggested by Merritt et al. ${ }^{10}$ The three ingredients were mixed together at the same temperature with a kitchen blender (Philips HR1363) at high speed for a minute. Samples of $40 \mathrm{ml}$ with $10 \%, 30 \%$, and $60 \%$ lipid-to-water ratio were prepared. Half of each solution was poured in separate containers to ultrasonicate the content. An ultrasonication probe (IKA T25 digital Ultra-Turrax) was used to reduce the particle size and thereby to change the scattering properties of the emulsions. Thus, six samples were prepared in total, with three different lipid-to-water ratios and different particle size distributions.

The particle size distribution (PSD) of each of the six samples was measured with an apparatus based on the Coulter principle (Multisizer 3 Coulter counter from Beckman Coulter). This apparatus could not resolve submicron particle sizes due to the limited dynamic range of the system. The PSD of the six emulsions we prepared in the lab is shown in Fig. 4. The first row of figures corresponds to the measurements performed on the samples that were mixed with the kitchen blender. The second row corresponds to the samples that were ultrasonicated. By comparing the PSD of the ultrasonicated with the nonultrasonicated samples for each of the various lipid-to-water ratios, it appears that the maximum of 


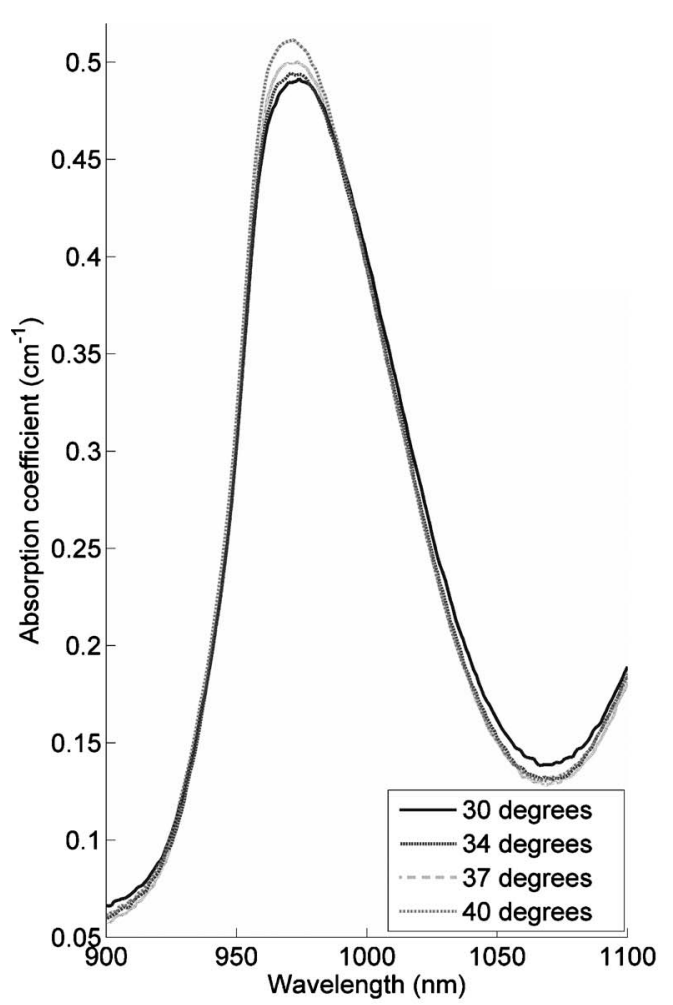

(a)

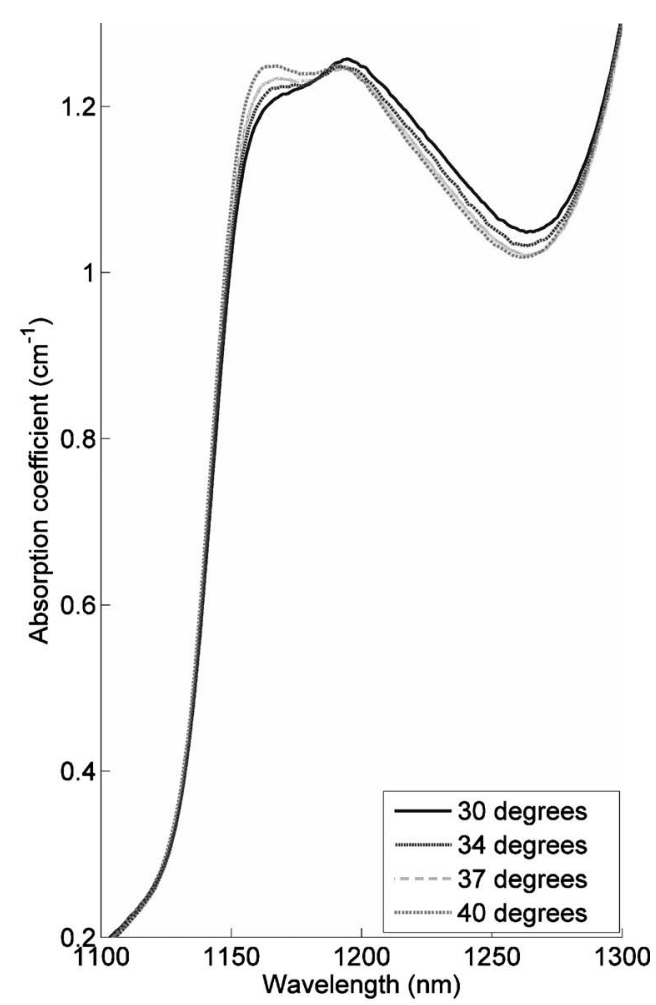

(b)

Fig. 2 Absorption coefficient of water at different temperatures for wavelength ranges (a) 900 to $1100 \mathrm{~nm}$ and (b) 1100 to $1300 \mathrm{~nm}$. The solid black line, black dotted line, gray dashed line, and gray dotted lines correspond to measurements at $30,34,37$, and $40{ }^{\circ} \mathrm{C}$, respectively.

the distribution shifts to the smaller particle size as expected. However, the standard deviation of the particle size is higher for lower lipid content, so if one wants to obtain a more uniform PSD for low lipid content samples, it is necessary to use other techniques.

\section{Results and Discussion}

Figure 5 and 6 show the measured spectra at a temperature around $30^{\circ} \mathrm{C}$ of the different lipid and water emulsions (symbol curves) and the corresponding best fits (solid line curves). The presence of lipid in the sample is correlated with the narrow peak at $1211 \mathrm{~nm}$, which is an absorption signature of lipid. Moreover, for the sample that contains more lipid than water, the 930-nm absorption peak of lipid is clearly visible in the measured spectrum. Above $1400 \mathrm{~nm}$, the intensity is null, and this is due to the very high absorption of water above this specific wavelength given the fiber distance separation.

The estimated parameters for each phantom emulsion are displayed in Table 1, and the variances of the fitted values derived from the calculation of the covariance matrix are displayed between brackets next to the estimated values. The estimation of the lipid-to-water ratio is within $5 \%$ error from the true value.

The scattering parameter increased with the amount of lipid in the sample. This was expected, since the lipid is the only scatterer in the various samples. The estimated slope value varied with respect to the scatterer size. ${ }^{4}$ From the estimated parameters for the slope, it appeared that the slope for the ultrasonicated samples was greater than for the nonultrasonicated samples. This observation correlates with the PSD measurements as discussed in the beginning of this sectioni.e., high slope values for samples with small average particles size.

In summary, we have shown with the set of custom-made phantoms that we could estimate the amount of water and lipid for different reduced scattering profiles from spectroscopic measurements with our optical probe using a fit to a diffusion approximation model. The following step was to complement the validation with a phantom study with a wider range of lipid-to-water ratios.

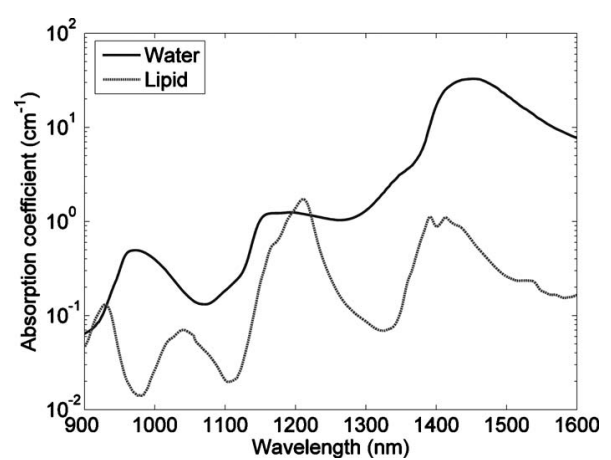

Fig. 3 Absorption spectra of water (solid line) and fat (dotted line) between 900 and $1600 \mathrm{~nm}$ 


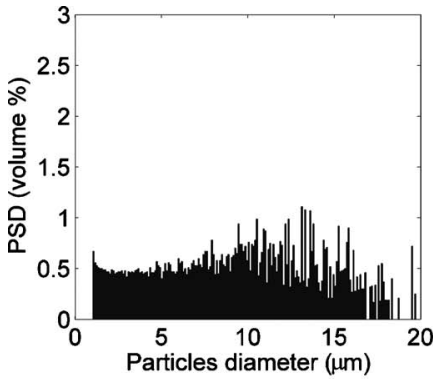

(a)

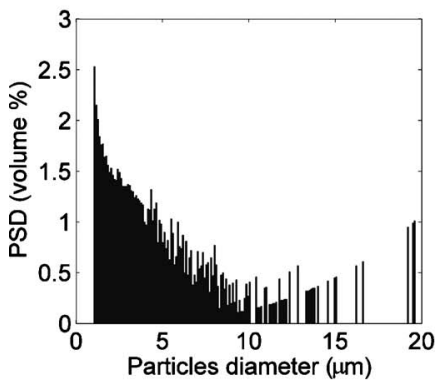

(d)

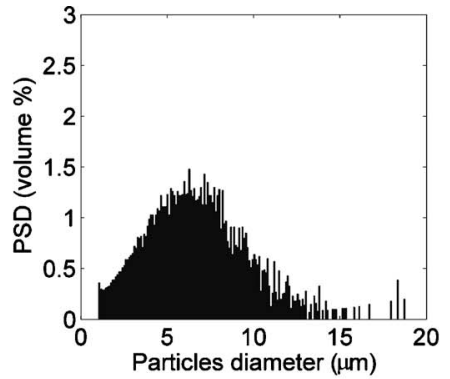

(b)

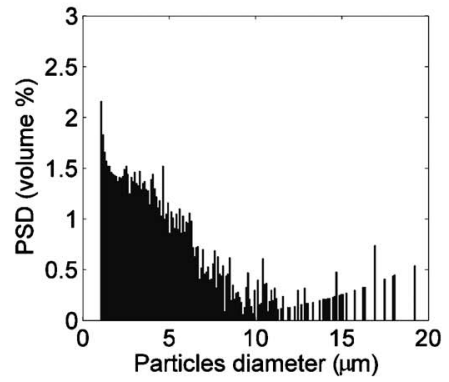

(e)

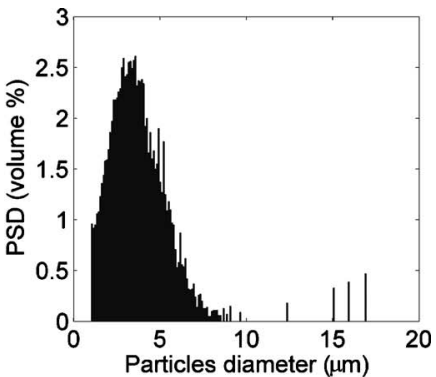

(c)

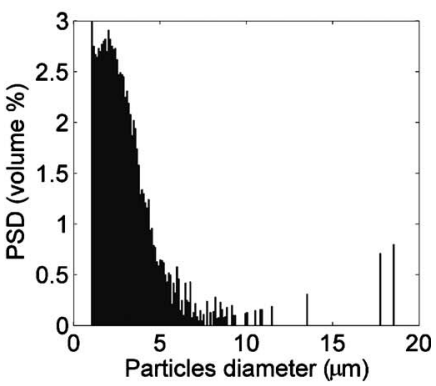

(f)

Fig. 4 Particle size distribution (PSD) measurements of the emulsions. The top row graphs show the PSD of the emulsions that were blended with a kitchen blender for the $10 \%$ (a), 30\% (b), and 60\% (c) lipid-to-water ratio, respectively. The bottom row shows the PSD of the same emulsions after applying an ultrasonic mixing for the $10 \%$ (d), 30\% (e), and 60\% (f) lipid-to-water ratio, respectively.

Measurements on commercial lipid-water samples such as butter and margarine were also performed at room temperature in order to further validate our method and to highlight the difference in the spectra for a wider range of lipid-towater ratios. Butter samples with $25 \%, 35 \%, 60 \%$, and $83 \%$ lipid content and a $40 \%$ lipid content margarine sample were also measured. A suspension of $320 \mathrm{mg}$ of $\mathrm{BaSO}_{4}$ in $20 \mathrm{ml}$ of water was prepared so that we had a light scattering sample without lipid in order to mimic muscle-like tissue with $0 \%$ lipid.

Figure 7 depicts the different measurements of the commercial butters (symbol curves) and the respective best fits (solid line curves). It is important to notice that the absorption spectra of lipid and water both have an absorption peak around 1200-nm wavelength but that the widths of these peaks are different. The lipid peak is much narrower than the

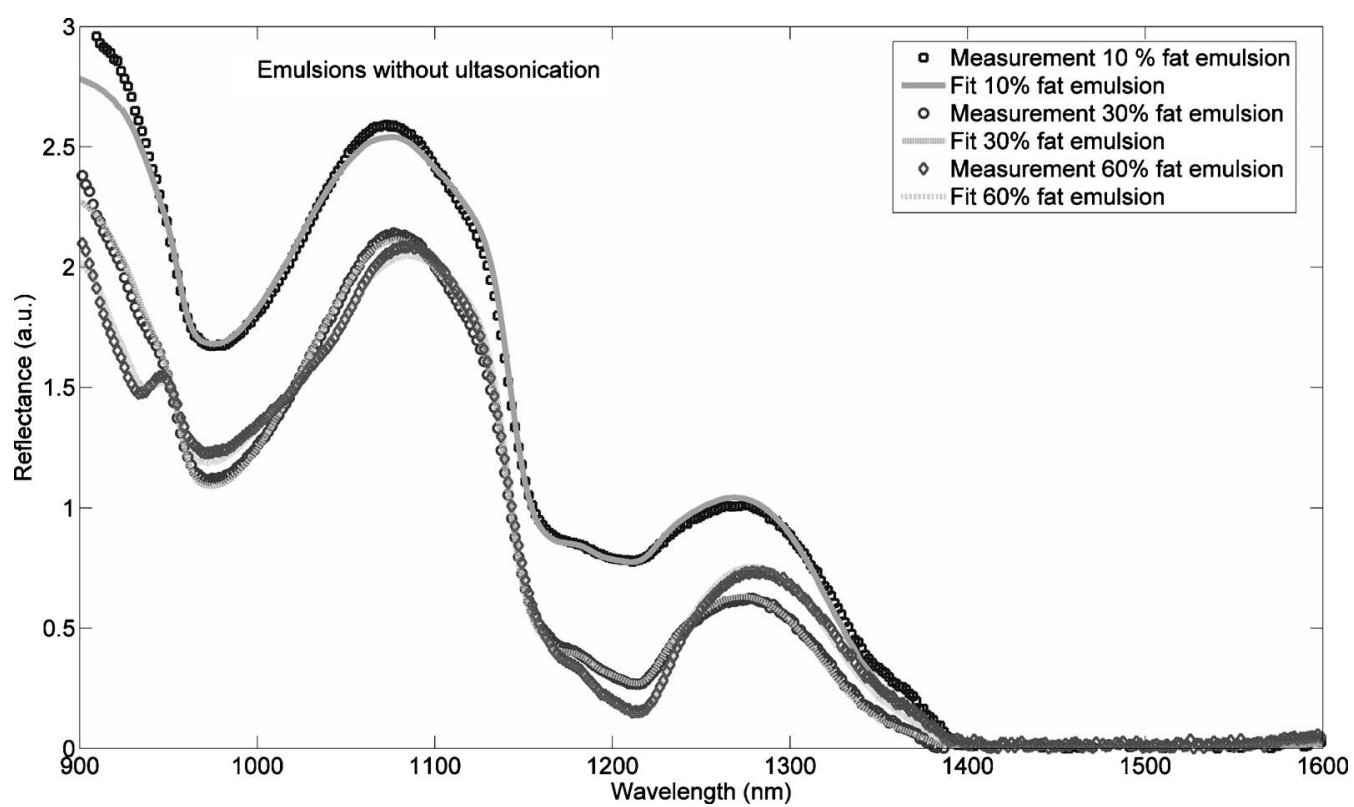

Fig. 5 Results of the fitting (solid line curves) on the measured spectra (symbol curves) of the different emulsions mixed with a blender. 


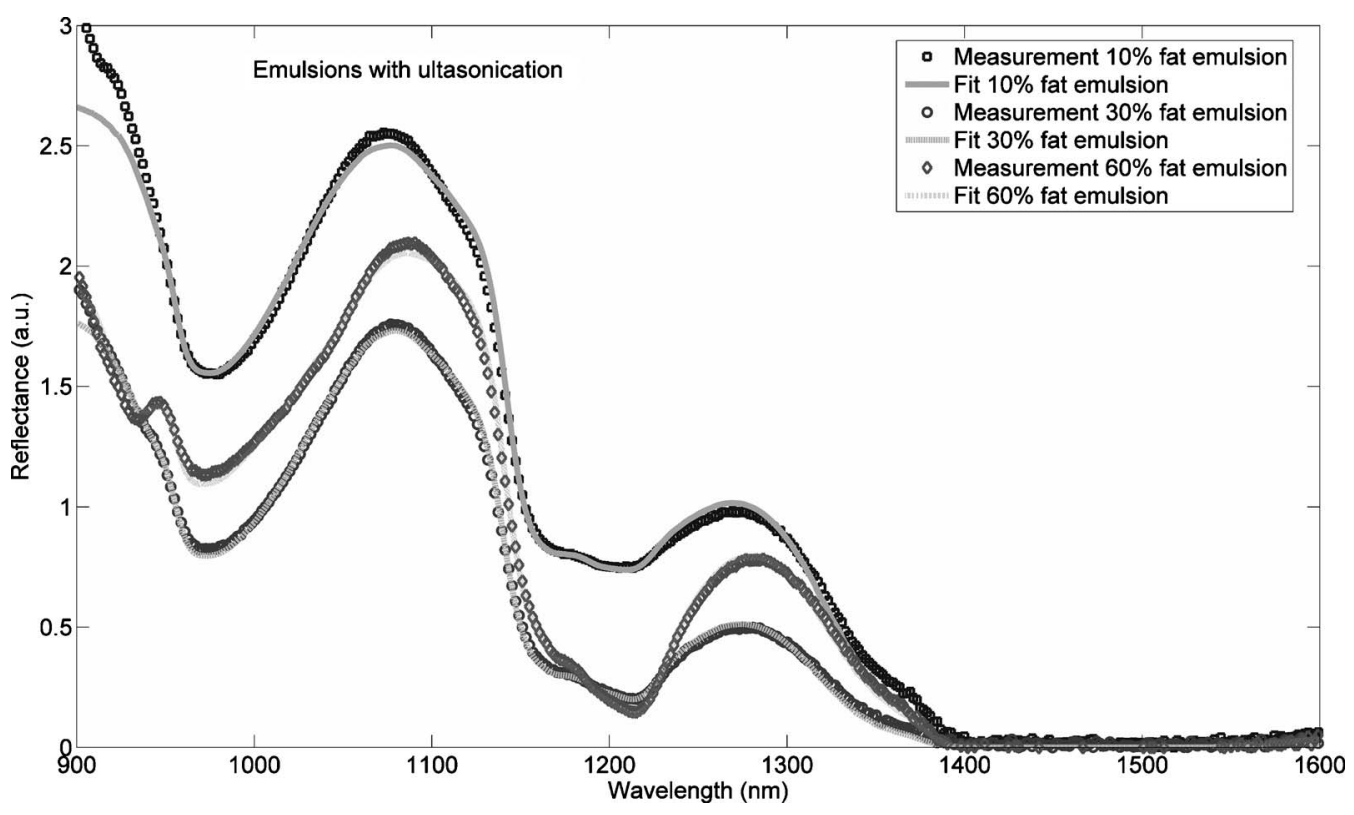

Fig. 6 Results of the fitting (solid line curves) on the measured spectra (symbol curves) of the different emulsions mixed with a blender; the sample preparation included an ultrasonication process.

water peak. For increasing concentration of lipid with respect to water, the measurements depicted in Fig. 7 show a narrowing of the peak in the spectra in the vicinity of $1200 \mathrm{~nm}$.

The fitting routine was performed on the measurements to estimate the amount of lipid and to compare it with the value written on the packages of the commercial samples. The results from the fits are summarized in Table 2 and reveal that with our method, it is possible to retrieve the amount of lipid with a deviation below $5 \%$ in comparison with the amount of lipid indicated on the commercial packages.

Several reasons can explain the deviations that were encountered and also the wide range of scattering between the different commercial samples. The fact that the absorption spectrum derived from beef fat was used as a reference absorption spectrum in the fitting routine is potentially a source of error, since it is a different kind of fat than in butters. Butter, margarine, and animal fats (e.g., lard) are known to be composed of different kind of fats, which are divided into three families: saturated fat, monounsaturated fat, and polyunsaturated fat. Butter is mainly composed of saturated fat (typically $66 \%$ saturated, $30 \%$ monounsaturated, and $4 \%$ polyun- saturated fat), whereas margarine is mainly composed of monounsaturated and polyunsaturated fat (typically $21 \%$ saturated, $46 \%$ monounsaturated, and $33 \%$ polyunsaturated fat), and animal fat of saturated and monounsaturated fat (typically $41 \%, 47 \%$, and $12 \%$ of respectively saturated, monounsaturated, and polyunsaturated fat). Figure 8 depicts the absorption coefficient of $100 \%$ lipid-content oil composed of $10 \%$ saturated, 30\% monounsaturated, and $60 \%$ polyunsaturated fat and the beef fat. One major difference is the presence of an extra absorption peak at $1170 \mathrm{~nm}$ and a slight difference in the absolute value of the absorption. The most optimal fit would be obtained by using the same type of fat as in each commercial sample to estimate the amount of lipid in the various commercial samples. Therefore, we decided to use only the absorption coefficient from the beef fat as the reference for lipid in the fitting. The more unsaturated the fat is, the larger the molecule. As a result, the scattering can vary significantly from sample to sample, depending on the distribution of the different types of fat in the sample (e.g., indus-

Table 1 Estimation of the optical properties of the custom emulsions without and with ultrasonication.

\begin{tabular}{|c|c|c|c|c|c|c|}
\hline \multirow{2}{*}{$\begin{array}{l}\text { Lipid-to-water-ratio } \\
\text { Ultrasonication }\end{array}$} & \multicolumn{2}{|c|}{$10 \%$} & \multicolumn{2}{|c|}{$30 \%$} & \multicolumn{2}{|c|}{$60 \%$} \\
\hline & Yes & No & Yes & No & Yes & No \\
\hline Water (\%) & $86.5( \pm 2.2)$ & $86.7( \pm 2.6)$ & $70.1( \pm 2.2)$ & $69.3( \pm 1.6)$ & $41.0( \pm 1.2)$ & $41.7( \pm 1.0)$ \\
\hline Lipid (\%) & $11.5( \pm 1.6)$ & $10.8( \pm 1.9)$ & $29.7( \pm 2.4)$ & $30.0( \pm 1.8)$ & $58.2( \pm 2.3)$ & $57.8( \pm 1.8)$ \\
\hline$\mu_{s}^{\prime}(1200 \mathrm{~nm})\left(\mathrm{cm}^{-1}\right)$ & $26.3( \pm 0.4)$ & $29.5( \pm 0.5)$ & $64.2( \pm 1.0)$ & $72.3( \pm 0.8)$ & $84.4( \pm 1.0)$ & $87.1( \pm 0.9)$ \\
\hline$b$ & $0.06( \pm 0.06)$ & $0.29( \pm 0.07)$ & $0.40( \pm 0.06)$ & $0.70( \pm 0.04)$ & $0.56( \pm 0.04)$ & $0.87( \pm 0.04)$ \\
\hline
\end{tabular}




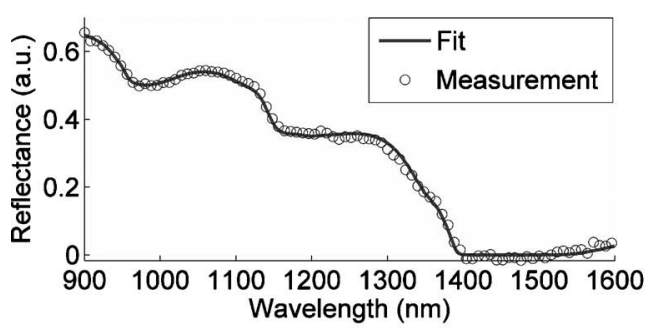

(a)

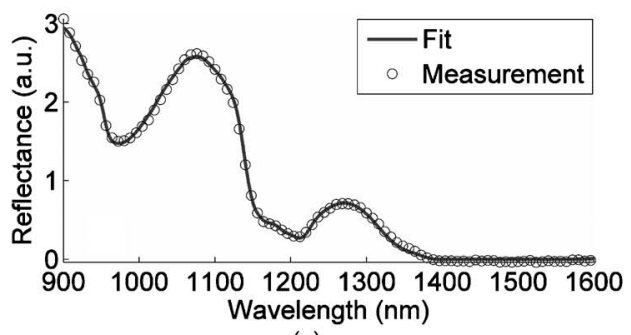

(c)

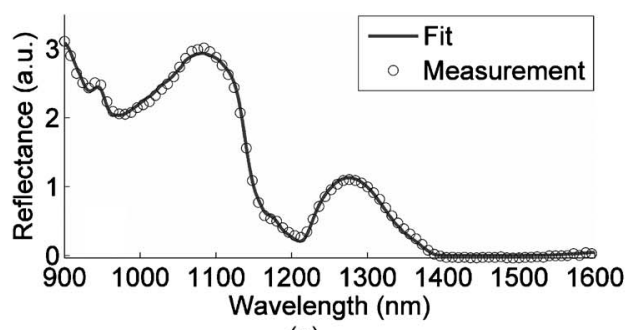

(e)

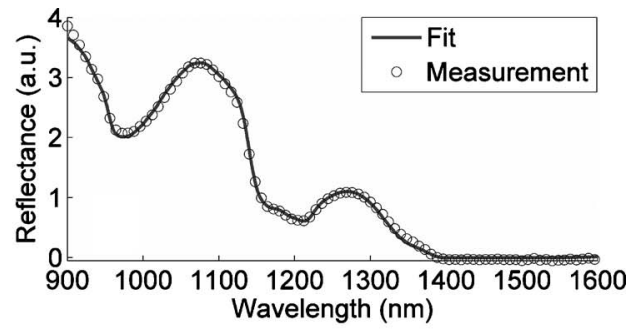

(b)

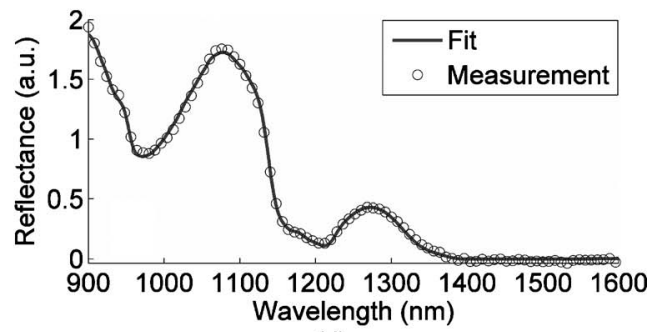

(d)

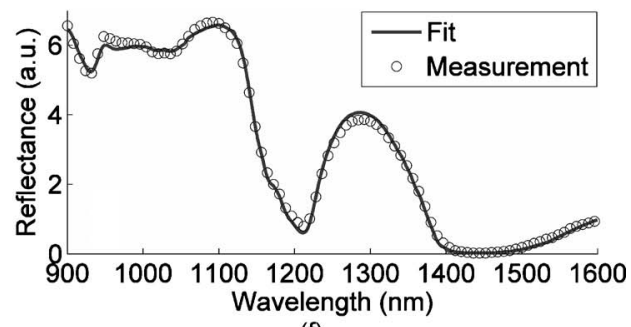

(f)

Fig. 7 Fits (solid line curves) to the measured spectra (data point curves) of the solution of $\mathrm{BaSO}_{4}$ in water (a) and the 25\% (b), 35\% (c), 40\% (d), $60 \%(\mathrm{e})$, and $83 \%$ (f) lipid content commercial butters and margarine.

trial margarine is composed of droplets of 5 to 10 microns diameter size).

In the case of the custom emulsions, it can clearly be seen that the fitting curve between 900 and $1000 \mathrm{~nm}$ is not accurate compared to the rest of the wavelength range. This is mainly due to the temperature of the sample. The PSD measurements were done just before the actual acquisition of the spectra, and thus the temperature of the samples was not constant. Several studies, such as Ref. 24, showed that the absorption coefficient of water is sensitive to temperature. Thus, the effect of temperature on the absorption coefficient of water is important to take into consideration.

Figure 9 shows ex vivo measurements (symbol curves) of excised tissue from a pig and their respective fit curves (solid line curves). The measurements were performed on subcutaneous fat, muscle, visceral fat, and white matter from the spinal cord by inserting the tip of the probe in the tissue. In Table 3, the estimated parameters for each tissue are summarized. Due to the fact that for the wavelength range of study, hemoglobin and oxygenated hemoglobin have their highest

Table 2 Estimation of the optical properties of the $\mathrm{BaSO}_{4}$ in water (A), 25\% lipid content butter (B), 35\% lipid content butter (C), 40\% lipid content margarine (D), 60\% lipid content butter (E), and 83\% lipid content butter (F).

\begin{tabular}{lcccccc}
\hline & \multicolumn{5}{c}{ Sample } \\
\cline { 2 - 6 } & $\mathrm{A}$ & $\mathrm{B}$ & $\mathrm{C}$ & $\mathrm{D}$ & $\mathrm{E}$ & $\mathrm{F}$ \\
\hline Water $(\%)$ & $93.6( \pm 2.5)$ & $74.6( \pm 1.5)$ & $63.9( \pm 1.1)$ & $57.3( \pm 1.1)$ & $36.1( \pm 0.8)$ & $13.8( \pm 0.5)$ \\
Lipid $(\%)$ & $0( \pm 4.6)$ & $25.2( \pm 1.7)$ & $36.0( \pm 1.6)$ & $42.7( \pm 1.8)$ & $63.7( \pm 2.0)$ & $85.4( \pm 2.5)$ \\
$\mu_{s}^{\prime}(1200 \mathrm{~nm})\left(\mathrm{cm}^{-1}\right)$ & $1.7( \pm 0.5)$ & $43.7( \pm 0.4)$ & $68.5( \pm 0.6)$ & $94.9( \pm 0.9)$ & $75.0( \pm 0.7)$ & $53.7( \pm 0.6)$ \\
$b$ & $0.55( \pm 0.03)$ & $0.01( \pm 0.03)$ & $0.03( \pm 0.02)$ & $0.33( \pm 0.02)$ & $0.09( \pm 0.03)$ & $0.01( \pm 0.03)$ \\
\hline
\end{tabular}




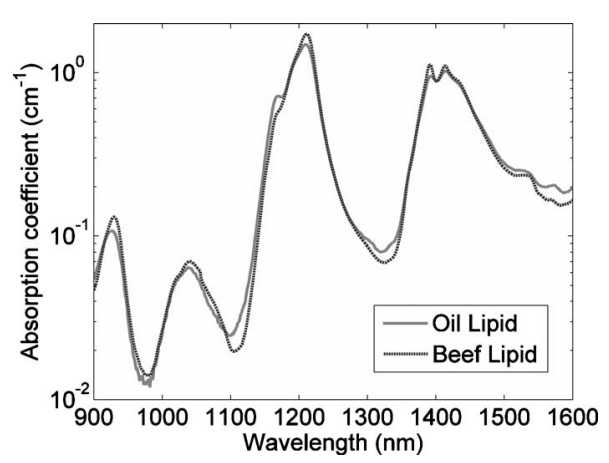

Fig. 8 Comparison between two types of lipids: the dotted line corresponds to the beef lipid, whereas the solid line curve corresponds to $100 \%$ lipid oil (sunflower oil).

absorption values (roughly $2 \mathrm{~cm}^{-1}$ ) between 900 and $1000 \mathrm{~nm}$, whereas water is the dominant absorber above $1000 \mathrm{~nm}$, as pinpointed in Ref. 25, we did not include these two absorbers in the fitting routine. Our ex vivo tissue samples did not contain more than $1 \%$ of total blood volume fraction, and thus hemoglobin did not have any influence on the fit parameters.

The estimated values are in agreement with those published from previous studies. ${ }^{26-29}$ The composition in lipid and water of the subcutaneous fat was defined in Ref. 28 as being of $14 \%$ water and $86 \%$ fat, which is in perfect agreement with our reported result. The estimation of water and from the muscle measurement is around $75 \%$, which is within the range of $60 \%$ to $80 \%$ of water in muscle, whereas the

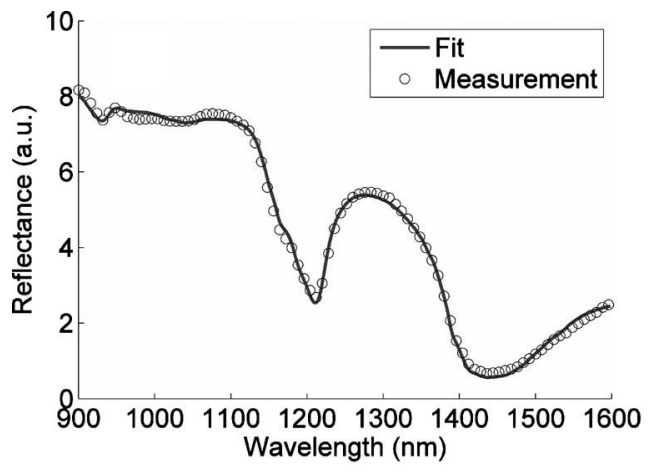

(a)

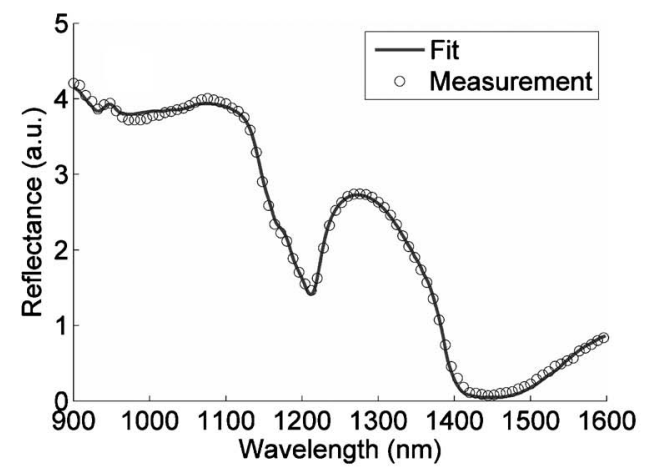

(c) estimated lipid content is roughly $2 \%$, within the $0 \%$ to $8 \%$ range. ${ }^{29}$ In visceral fat, the estimation of lipid is $74 \%$, within the lipid content range of $60 \%$ and $90 \%$ in adipose tissue. ${ }^{29}$ White matter is mainly axons surrounded with myelin; this physiological property is also in agreement with our finding from the acquired spectrum. From the estimation of the optical properties, it follows that white matter contains about $25 \%$ of lipid and the rest is water, which is slightly above the reported 20\% (Ref. 29). The scattering amplitudes that are estimated from the measured tissues are within the range of values available in Ref. 27. References 6 and 26, respectively, present the scattering of different tissues and fatty breasts, which are comparable to our results. The scattering slope values estimated are within the range of 0.18 to 2.84 described in Ref. 26.

The absorption and reduced scattering coefficient can be computed thanks to the relation described in Eq. (3) and the power law function, respectively. Figure 10 shows the optical properties for each of the ex vivo tissue measurements that were computed once the fit parameters in Table 3 were obtained. Although the reduced scattering coefficient above $1350 \mathrm{~nm}$ is lower than the absorption coefficient, it is still possible to estimate the proper volume fraction of water and lipid. In particular, the fact that water and lipid have sharp peaks in the 900- to 1000-nm wavelength range and around $1200 \mathrm{~nm}$ makes the estimation of the amount of water and lipid in the full range of 900 to $1600 \mathrm{~nm}$ still possible. For wavelengths below $1350 \mathrm{~nm}$, the reduced scattering coefficient is larger than the absorption coefficient. Since the reduced scattering coefficient is modeled by a power law for the

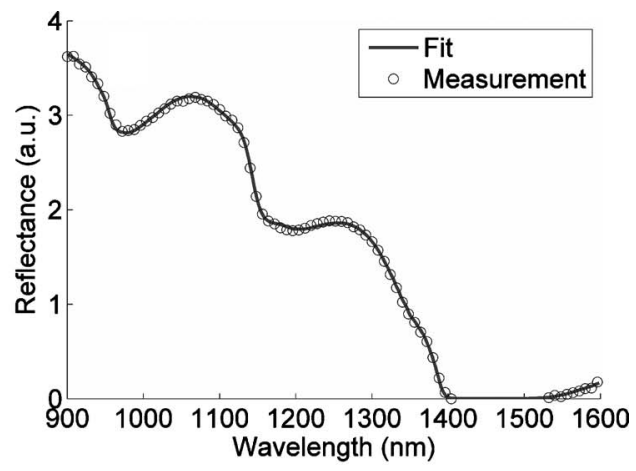

(b)

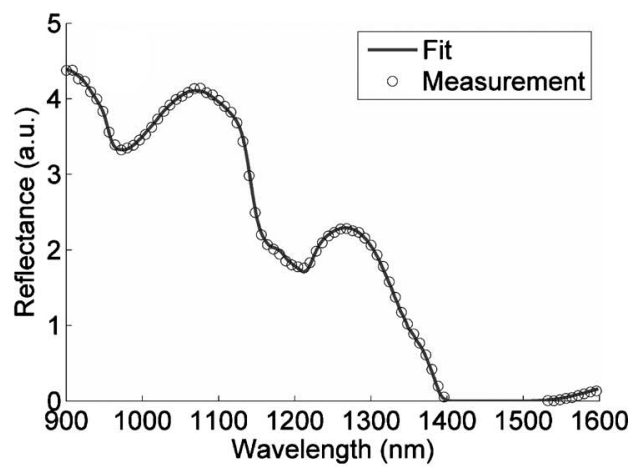

(d)

Fig. 9 Fits (solid line curves) to the ex vivo tissue measurements (data point curves) of subcutaneous fat (a), muscle (b), visceral fat (c), and white matter $(\mathrm{d})$. 
Nachabé et al.: Estimation of lipid and water concentrations in scattering media...

Table 3 Estimated optical properties of various swine tissues measured ex vivo.

\begin{tabular}{lcccc}
\hline & Subcutaneous fat & Muscle & Visceral fat & White matter \\
\hline Water $(\%)$ & $12.2( \pm 0.5)$ & $74.6( \pm 2.2)$ & $24.9( \pm 0.9)$ & $76.8( \pm 1.5)$ \\
Lipid $(\%)$ & $89.6( \pm 5.0)$ & $1.9( \pm 1.2)$ & $74.0( \pm 4.1)$ & $26.4( \pm 1.3)$ \\
$\mu_{s}^{\prime}(1200 \mathrm{~nm})\left(\mathrm{cm}^{-1}\right)$ & $4.5( \pm 0.6)$ & $3.1( \pm 0.2)$ & $5.3( \pm 0.7)$ & $6.0( \pm 0.3)$ \\
$b$ & $1.76( \pm 0.21)$ & $1.17( \pm 0.08)$ & $1.92( \pm 0.33)$ & $1.58( \pm 0.24)$ \\
\hline
\end{tabular}

whole wavelength range, the reduced scattering amplitude and slope constrained the fit, and thus it is possible to evaluate the optical properties, despite the fact that absorption can be higher than the reduced scattering above $1350 \mathrm{~nm}$. However, for low-lipid content tissues with low reduced scattering, care must be taken, since the method may become inaccurate. If the fit deviates from the measurements for wavelengths above $1350 \mathrm{~nm}$, this would be a sign that the applied model is no longer valid. For high water content tissue, the signal can become null, given our fiber distance separation, which also allows for a more stable fit. One can notice that around $1200 \mathrm{~nm}$, the absorption coefficient of water and lipid have roughly the same absolute values, making this ratio less sensitive to errors in the fit model.

\section{Conclusion}

To accurately estimate water and lipid content in a small volume (e.g., a few $\mathrm{mm}^{3}$ ), a higher extinction is preferable than that of the commonly used 900- to 1000-nm absorption peaks. In the wavelength range from 900 to $1600 \mathrm{~nm}$, one finds more absorption peaks with higher absorption coefficients. We performed accurate measurements of the water and lipid extinction coefficient to allow us to obtain a reliable estimation of these two chromophores in mixtures.

We have demonstrated in this study that we can accurately estimate the amount of lipid and water with a diffuse optical spectroscopy technique in the near infrared for wavelengths in the range of 900 to $1600 \mathrm{~nm}$. The phantom study proved that

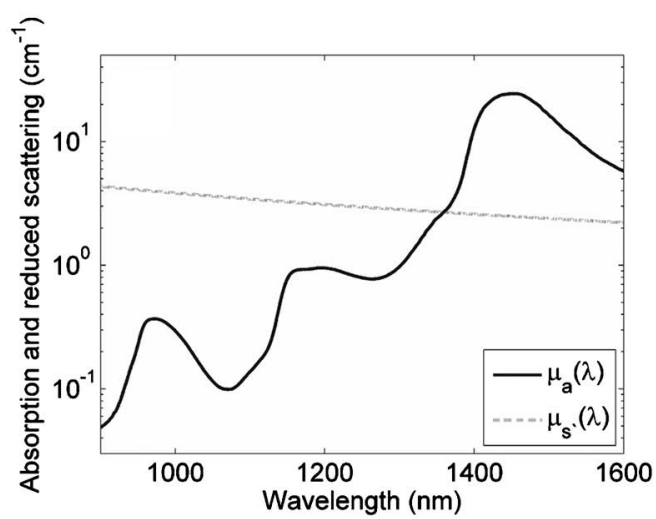

(b)

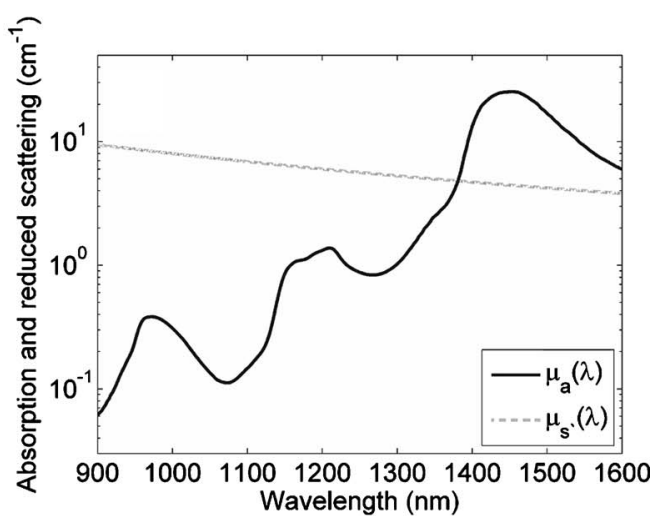

(d)

Fig. 10 Absorption and reduced scattering coefficients of the ex vivo tissue measurements of subcutaneous fat (a), muscle (b), visceral fat (c), and white matter $(d)$. 
we can estimate, within less than 5\% error, the amount of lipid and water in the various samples that were prepared. Furthermore, by applying ultrasonication to our phantoms to shrink the particle size, we have seen that the slope of the reduced scattering increased without effecting the estimation of water and lipid content. The validation of our method was completed with the estimation of the concentration of lipid in commercial food samples with known lipid content.

This phantom study is complemented by ex vivo measurements and analysis that provide information about the optical properties of different tissues. The amount of lipid and water will prove to be important for discriminating healthy and cancerous tissue. With our method, we could provide real-time clinical feedback to physicians on the amount of lipid and water in tissue with the goal to classify between normal, malignant, and benign samples when acquiring spectra from fiber-tissue probes.

\section{Acknowledgments}

We are grateful to Roy Le Clercq and Marcel Böhmer from Philips Research for their help in the experiments. We also thank Gerald Lucassen, Martin Vernhout, Jean Schleipen, and Walter Bierhoff from Philips Research and Arjen Amelink from Erasmus Medical Center for their collaboration. This work is supported by a European Commission Marie Curie Contract MEST-CT-2004-007832.

\section{References}

1. G. Zonios, L. T. Perelman, V. M. Backman, R. Manoharan, V. D Fitzmaurice, J. Van Dam, and M. S. Feld, "Diffuse reflectance spectroscopy of human adenomatus colon polyps in vivo," Appl. Opt. 38 6628-6637 (1999)

2. J. R. Mourant, I. J. Bigio, J. Boyer, T. M. Johnson, and J. Lancey, "Elastic scattering spectroscopy as a diagnostic for differentiating pathologies in the gastrointestinal tract: preliminary testing," $J$. Biomed. Opt. 1, 192-199 (1996)

3. M. P. L. Bard, A. Amelink, V. N. Hegt, W. J. Graveland, H. J. C. M. Sterenborg, H. C. Hoogsteden, and J. G. J. V. Aerts, "Measurement of hypoxia-related parameters in bronchial mucosa by use of optical spectroscopy," Am. J. Respir. Crit. Care Med. 171, 1178-1184 (2005).

4. G. Zonios and A. Dimou, "Light scattering spectroscopy of human skin in vivo," Opt. Express 17, 1256-1267 (2009).

5. G. Zonios, J. Bykowski, and N. Kollias, "Skin melanin, hemoglobin, and light scattering properties can be quantitatively assessed in vivo using diffuse reflectance spectroscopy," J. Invest. Dermatol. 117, 1452-1457 (2001).

6. A. Amelink, O. P. Kaspers, H. J. C. M. Sterenborg, J. E. Van der Wal, J. L. N. Roodenburg, and M. J. H. Witjes, "Non-invasive measurement of the morphology and physiology of oral mucosa by use of optical spectroscopy," Oral Oncol. 44, 65-71 (2008).

7. A. E. Cerussi, N. Shah, D. Hsiang, A. Durkin, J. Butler, and B. J. Tromberg, "In vivo absorption, scattering of 58 malignant breast tumors determined by broadband diffuse optical spectroscopy," $J$. Biomed. Opt. 11(4), 044005 (2006).

8. A. Pifferi, P. Taroni, A. Torricelli, F. Messina, R. Cubeddu, and G. Danesini, "Four wavelength time-resolved optical mammography in the 680-980 nm range," Opt. Lett. 28, 1138-1140 (2003).

9. R. Michels, F. Foschum, and A. Kienle, "Optical properties of fat emulsion," Opt. Express 16, 5907-5925 (2008).

10. S. Merritt, G. Gulsen, G. Chiou, Y. Chu, C. Deng, A. E. Cerussi, A. J. Durkin, B. J. Tromberg, and O. Nalcioglu, "Comparison of water and lipid content measurements using diffuse optical spectroscopy and MRI in emulsion phantoms," Technol. Cancer Res. Treat. 2, 563 569 (2003).

11. R. R. Anderson, W. Farinelli, H. Laubach, D. Manstein, A. N. Yaroslavsky, J. Gubelli, K. Jordan, G. R. Neil, M. Shinn, W. Chandler, G. P. Williams, S. V. Benson, D. R. Douglas, and H. F. Dylla, "Selective photothermolysis of lipid-rich tissues: a free electron laser study," Lasers Surg. Med. 38, 913-919 (2006).

12. A. N. Bashkatov, E. A. Genina, V. I. Kochubey, and V. V. Tuchin, "Optical properties of human skin, subcutaneous and mucous tissues in the wavelength range from 400 to $2000 \mathrm{~nm}$, , J. Phys. D 38, 2543 2555 (2005).

13. T. L. Roy and S. N. Thennadil, "Optical properties of human skin in the near infrared wavelength range of 1000 to $2200 \mathrm{~nm}$," J. Biomed. Opt. 6, 167-176 (2001).

14. T. J. Farrell, M. S. Patterson, and B. Wilson, "A diffusion theory model of spatially resolved, steady-state diffuse reflectance for the non-invasive determination of tissue optical properties," Med. Phys. 19, 879-888 (1992).

15. A. Amelink, T. Christiaanse, and H. J. C. M. Sterenborg, "Effect of hemoglobin extinction coefficient spectra on optical spectroscopic measurements of blood oxygen saturation," Opt. Lett. 34(10), 15251527 (2009).

16. P. R. Bargo, S. A. Prahl, T. T. Goodell, R. A. Sleven, G. Koval, G. Blair, and S. L. Jacques, "In vivo determination of optical properties of normal and tumor tissue with white light reflectance and an empirical light transport model during endoscopy," J. Biomed. Opt. 10, 034018 (2005).

17. G. Zonios, I. Bassukas, and A. Dimou, "Comparative evaluation of two simple diffuse reflectance models for biological tissue applications," Appl. Opt. 47, 4965-4973 (2008).

18. J. Sun, K. Fu, A. Wang, A. W. H. Lin, U. Utzinger, and R. Drezek, "Influence of fiber optic probe geometry on the applicability of inverse models of tissue reflectance spectroscopy: computation models and experimental measurements," Appl. Opt. 45, 8152-8162 (2006).

19. R. Reif, O. A'Amar, and I. J. Bigio, "Analytical model of light reflectance for extraction of the optical properties in small volumes of turbid media," Appl. Opt. 46, 7317-7328 (2007).

20. A. Amelink, H. J. C. M. Sterenborg, M. P. L. Bard, and S. A. Burgers, "In vivo measurement of the local optical properties of tissue by use of differential path-length spectroscopy," Opt. Lett. 29, 10871089 (2004).

21. A. Amelink, D. J. Robinson, and H. J. C. M. Sterenborg, "Confidence interval on fit parameters derived from optical reflectance spectroscopy measurements," J. Biomed. Opt. 13, 054044 (2008).

22. R. L. P. van Veen, H. J. C. M. Sterenborg, A. Pifferi, A. Torricelli, E. Chikoidze, and R. Cubeddu, "Determination of visible near-IR absorption coefficients of mammalian fat using time- and spatially resolved diffuse reflectance and transmission spectroscopy," J. Biomed. Opt. 10, 054004 (2005).

23. G. M. Hale and M. R. Querry, "Optical constants of water in the 200-nm to 200-micrometer wavelength region," Appl. Opt. 12, 555563 (1973)

24. K. Buijs and G. R. Choppin, "Near-infrared of the structure of water," J. Chem. Phys. 39, 2035-2041 (1963).

25. A. Roggan, M. Friebel, K. Dorchel, A. Hahn, and G. Muller, "Optical properties of circulating human blood in the wavelength range of 400-2500 nm," J. Biomed. Opt. 4, 36 (1999).

26. R. L. P. van Veen, W. Verkruysse, and H. J. C. M. Sterenborg, "Diffuse reflectance spectroscopy from 500 to $1060 \mathrm{~nm}$ by correction for inhomogenously distributed absorbers," Opt. Lett. 27, 246-248 (2002).

27. V. V. Tuchin, "Tissue optics: light scattering methods and instruments for medical diagnosis," in SPIE Tutorial Texts in Optical Engineer ing, 2nd ed., pp. 170-191, SPIE Press, Bellingham, WA (2007).

28. J. Wang and R. N. Pierson, "Disparate hydration of adipose and lean tissue require a new model for body water distribution in man," $J$. Nutr. 106, 1687-1693 (1976).

29. F. A. Duck, Physical Properties of Tissue: A Comprehensive Reference Book, Academic Press, Harcourt Brace Jovanovich, New York (1990). 